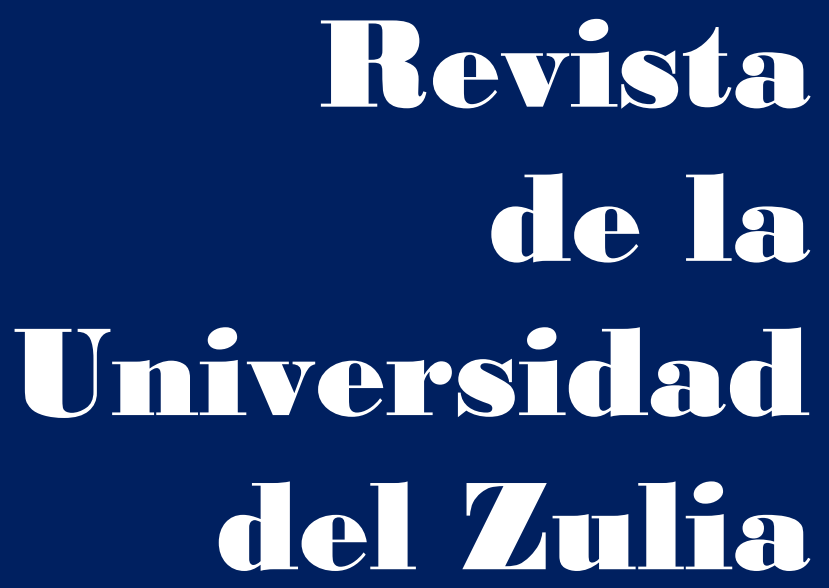

Fundada en 1947

por el Dr. Jesús Enrique Lossada

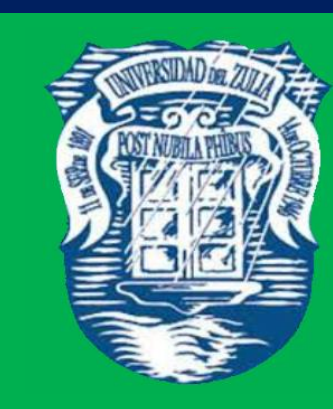

Ciencias del

Agrad,

Ingemiería

y Tecinología

\section{Aกัต 13 No $\mathbf{3 6}$} Enero - Abril 2022

Tercera Épaca

Maracailbo-Venezuela 
REVISTA DE LA UNIVERSIDAD DEL ZULIA. $3^{a}$ época. Año $13 \mathrm{~N}^{\circ}$ 36, 2022

Jhon More Calderón et al. // Caracterización física y sensorial de almendras de plantas de cacao ... 56-69

DOI: http://dx.doi.org/10.46925//rdluz.36.04

\title{
Caracterización física y sensorial de almendras de plantas de cacao elite (Theobroma cacao L.) en Bagua, Perú
}

\author{
Jhon More Calderón* \\ Armstrong Barnard Fernández Jeri** \\ Efraín Manuelito Castro Alayo*** \\ César Balcázar Zumaeta**** \\ Leoncio Hertz Fernández Jeri*****
}

RESUMEN

El trabajo de investigación tuvo como objetivo caracterizar física y sensorialmente las almendras de cacao (Theobroma cacao L.) de 20 plantas elites en fincas de los distritos Copallín y La Peca en la provincia Bagua, Perú. Se determinó la calidad física (índice de grano, peso de grano y prueba de corte) y sensorial (atributos básicos y específicos). Se obtuvo valores de humedad de las almendras (6,5 a 7,7\%) dentro los valores aceptables (menor a 8\%); el promedio de índice de grano y peso de grano indicaron un manejo agronómico y empleo de material genético adecuados; el índice de grano $(1,20$ a 1,80) en 18 árboles de cacao elites fueron valores comerciales. Sensorialmente los atributos básicos se acentuaron en las almendras de Copallín; mientras que los atributos floral, frutal y cacao resaltaron más en almendras de La Peca. Las almendras estudiadas son aptas para la obtención de chocolate de fino aroma.

PALABRAS CLAVE: Producto vegetal; producto agrícola; Economía agraria; cacao; Perú.

* Bachiller de la Facultad de Ingeniería y Ciencias Agrarias. Universidad Nacional Toribio Rodríguez de Mendoza de Amazonas, Perú. ORCID: https:/orcid.org/0000-0001-6047-0707. Email: hing.jaa@gmail.com.

** Instituto de Investigación, Innovación y Desarrollo para el Sector Agrario y Agroindustrial de la región Amazonas (IIDAA-Amazonas), Universidad Nacional Toribio Rodríguez de Mendoza de Amazonas, Perú. ORCID: https://orcid.org/0000-0001-9476-1078. E-mail: armstrong.fernandez@untrm.edu.pe

*** Instituto de Investigación, Innovación y Desarrollo para el Sector Agrario y Agroindustrial de la región Amazonas (IIDAA-Amazonas), Universidad Nacional Toribio Rodríguez de Mendoza de Amazonas, Perú. ORCID: https://orcid.org/0000-0003-4322-8980. E-mail: efrain.castro@untrm.edu.pe

****Instituto de Investigación, Innovación y Desarrollo para el Sector Agrario y Agroindustrial de la región Amazonas (IIDAA-Amazonas), Universidad Nacional Toribio Rodríguez de Mendoza de Amazonas, Perú. ORCID: https://orcid.org/0000-0002-3033-6440. E-mail: cesar.balcazar@untrm.edu.pe

****Escuela de Agronegocios. Post Grado. Universidad Nacional Agraria La Molina, Perú. ORCID: https://orcid.org/0000-0002-7863-0665. E-mail: leojeri@lamolina.edu.pe

El artículo presenta los resultados centrales de un Trabajo de Grado en la Universidad Nacional Toribio Rodríguez de Mendoza de Amazonas, Perú.

Recibido: 15/09/2021

Aceptado: 11/11/2021 


\section{Physical and sensory characterization of almonds from elite cacao plants (Theobroma cacao L.) in Bagua, Peru}

ABSTRACT

The objective of the research work was to physically and sensorially characterize the cacao almonds (Theobroma cacao L.) of 20 elite plants on farms in the Copallín and La Peca districts in Bagua province, Peru. Physical (grain index, grain weight and cutting test) and sensory quality (basic and specific attributes) were determined. Moisture values of the almonds (6.5 to $7.7 \%$ ) were obtained within acceptable values (less than $8 \%$ ); the average grain index and grain weight indicated adequate agronomic management and use of genetic material; the bean index (1.20 to 1.80) in 18 elite cacao trees were commercial values. Sensorially, the basic attributes were accentuated in the Copallín almonds; while the floral, fruity and cocoa attributes were stood out in La Peca almonds. The studied almonds are suitable for obtaining fine aroma chocolate.

KEYWORDS: Plant products; Agricultural products; Agricultural economics; cocoa; Peru.

Introducción

El cacao (Theobroma cacao L.) es nativo de regiones tropicales húmedas. Hay cuatro grupos genéticos comúnmente reconocidos: forastero, criollo, trinitario y un cuarto grupo referido a la variedad Nacional que crece en Ecuador. Los forasteros son los más cultivados por su alta resistencia a enfermedades; mientras que los trinitarios tienen resistencia a enfermedades y alta productividad, pero en sabor son de menor calidad para la producción de chocolates (Castro-Alayo et al. 2019; Ramos, et al. 2020). Los granos de cacao criollo resaltan debido a sus atributos sensoriales especiales de aroma floral y afrutado; mientras que los otras variedades abarcan a los granos de cacao forastero y su híbrido, trinitario (buena calificación), donde se aprecia que el sabor de cacao fino está siendo requerido por las industrias mundiales de chocolate de sabor fino; el atributo sensorial de calidad más importante durante su comercialización es el aroma del grano de cacao; este aroma está formado por compuestos volátiles que son percibidos en la cavidad nasal (Chire et al. 2016; Qin et al. 2017; Wickramasuriya \& Dunwell, 2018; Castro-Alayo, 2019).

La calidad de las almendras de cacao se debe básicamente a sus compuestos aromáticos, siendo el genotipo el factor de mayor relevancia; así mismo, las condiciones 
REVISTA DE LA UNIVERSIDAD DEL ZULIA. $3^{a}$ época. Año $13 \mathrm{~N}^{\circ}$ 36, 2022

Jhon More Calderón et al. /// Caracterización física y sensorial de almendras de plantas de cacao ... 56-69

DOI: http://dx.doi.org/10.46925//rdluz.36.04

agroclimáticas en las que se desarrolla el cultivo, los procesos de cosecha, fermentación, secado y procesamiento, son factores relevantes en la formación de componentes volátiles que definen la calidad del producto final. Es tendencia estandarizar la calidad en el manejo postcosecha en los granos que permitan realizar una serie de transformaciones físicas y químicas que permitan desarrollar su calidad exigida por el mercado y los agronegocios (Peláez et al. 2016; García \& Muñoz, 2017; Quevedo et al. 2018; Moreno-Martínez et al. 2019).

Debido a los requisitos exigidos de la calidad de las almendras de cacao, el estudio de cacao elites es primordial por sus características morfológicas y de productividad, así como se deben mejorar las semillas y plantaciones (Vera et al. 2019). Estos requisitos se expresan en términos de características físicas. Según García \& Muñoz (2017) y Quevedo et al. (2018), se deben considerar la apariencia, prueba de corte, índice de grano, humedad, tamaño del grano, mohos, insectos, enfermedades, entre otros.

En estudios previos sobre la calidad física del grano de cacao, García \& Muñoz (2017) y Gutiérrez \& Gonzales (2018) mencionan que los granos fermentados y secos de cacao criollo presentan colores castaños y cotiledones agrietados productos de un buen beneficio del cacao; comparativamente variedades como trinitario y forastero tienen colores pardo oscuro con cotiledones agrietados; además, el cacao criollo presenta más rendimiento en peso comparado con las otras variedades restantes; esto último se debe básicamente a un adecuado manejo postcosecha.

García \& Muñoz (2017), Machado et al. (2018) y Barrientos et al. (2019) afirman que la calidad sensorial del cacao es esencial para el consumo; los procesos postcosecha que se realizan en las almendras y posiblemente la presencia de polifenoles en su composición, determinan el perfil sensorial en el cacao con aroma y sabores amargo, ácido, astringente y a nueces.

En el presente trabajo de investigación se intenta caracterizar física y sensorialmente almendras de cacao (Theobroma cacao L.) élites, cultivados en la provincia de Bagua, región Amazonas, Perú. Con los resultados encontrados se busca seleccionar los plantones elites de cacao y cultivar en otras fincas de pequeños productores cacaoteros para obtener una alta producción y mejor calidad de grano exigido en la comercialización con el mercado externo. 
REVISTA DE LA UNIVERSIDAD DEL ZULIA. $3^{a}$ época. Año $13 \mathrm{~N}^{\circ}$ 36, 2022

Jhon More Calderón et al. /// Caracterización física y sensorial de almendras de plantas de cacao ... 56-69

DOI: http://dx.doi.org/10.46925//rdluz.36.04

1. Materiales y métodos

La investigación se llevó a cabo en las fincas cacaoteras de productores de los distritos de Copallín y La Peca, provincia de Bagua (Perú) en los años 2018 a 2020. Se recolectaron frutos maduros de plantas de cacao nativo elites de 20 fincas cultivadas entre los $888 \mathrm{msnm}$ y 1133 msnm; se consideraron muestras por triplicado, siendo codificadas con las iniciales de los nombres de los productores. Para la selección de árboles elites se tuvo en cuenta lo mencionado por Ballesteros (2011), como árboles mayores de 15 años de edad, el número de frutos que tenga el árbol 2 meses antes del pico de la cosecha sea mayor a 80 frutos por árbol, índices de mazorca por debajo de 18 por kilo de cacao seco, e índice de semillas mayores de 1,5 gramos por grano, además de alta tolerancia a plagas, enfermedades, estrés de sequía y humedad.

Para determinar la humedad se empleó la técnica descrita por Intriago et al. (2019), se pesó y trituró 10 gramos de almendras usando mortero hasta obtener un tamaño de partícula de hasta 5mm; luego, en una balanza de humedad se determinó la humedad por diferencias de pesos. Para el peso del grano se determinó el número de granos en 100 gramos y el peso promedio por grano (índice de grano-IG) según lo descrito por Vallejo et al. (2018); para IG se calculó la relación de peso de almendras entre número de almendras. Para la prueba de corte se siguió lo descrito por Vallejo et al. (2018), se seleccionaron al azar 50 almendras, después se cortó con un estilete por la mitad cada una de las almendras, siendo colocadas en una superficie plana con fondo de color blanco para tener mejor apreciación de la coloración de los granos cortados; posteriormente se clasificaron las almendras según su fermentación en los siguientes niveles: bien fermentados, levemente fermentados y pizarrosos.

\section{1.l. Calidad sensorial}

Para determinar el perfil sensorial se consideró la metodología descrita por Vallejo et al. (2018), con tal fin se pesó $300 \mathrm{~g}$ de cacao de cada muestra y se tostaron en horno de secado $\left(120^{\circ} \mathrm{C}, 25 \mathrm{~min}\right)$ por convección natural de aire. Luego se obtuvo el licor de cacao, con tal fin se procesaron en un molino, hasta obtener partículas entre 20-25 $\mu$ de tamaño. Los licores se colocaron en recipientes herméticos, rotulados con los datos de cada muestra y se almacenaron a $7^{\circ} \mathrm{C}$. Los principales atributos sensoriales en las almendras: básicos (dulce, amargo, astringente y ácido) y específicos (afrutado, cacao, floral, nueces y crudo) fueron 
REVISTA DE LA UNIVERSIDAD DEL ZULIA. $3^{a}$ época. Año $13 \mathrm{~N}^{\circ}$ 36, 2022

Jhon More Calderón et al. /// Caracterización física y sensorial de almendras de plantas de cacao ... 56-69

DOI: http://dx.doi.org/10.46925//rdluz.36.04

evaluados por catadores. Para la intensidad de degustación sensorial se tuvo como referencia la siguiente calificación y puntaje: ausente (0), bajo (l a 2), medio (3 a 5), alto (6 a 8) y muy fuerte (9 a 10).

\subsection{Análisis estadístico}

El análisis de las variables y datos de estudio se llevó a cabo empleando la estadística descriptiva. Se elaboraron tablas y figuras para ilustrar los datos. Asimismo, para la evaluación sensorial realizada se empleó análisis de componentes principales (PC) para analizar la dispersión de las variables cuantitativas agrupadas según sus atributos sensoriales de almendras de cacao elites.

\section{Resultados y discusión}

2.1. Determinación de las características físicas de almendras de cacao élites

Las características físicas de almendras de árboles de cacao elites se presentan en la Tabla 1. Se observa la humedad en las almendras del total de árboles elite (20), la cual fluctuó en un rango de 6,5\% a 7,7\%. Al respecto García \& Muñoz (2017), Guimac (2017) y Machado et al. (2018) establecen que el contenido de humedad del grano de cacao en la fermentación es de 60\% y luego al secarse puede llegar hasta 6\% al 7\%. Así mismo, los valores de 06 muestras estuvieron dentro del rango requerido (ABL-01, ABL-02, DTM-02, JBB-02, MRC08 y SCF-01) y las restantes fueron superior al 7\%, valores considerados como aceptables (menor al 8\%); estos valores bajos de humedad mantenida en las almendras favorecería a la preservación y comercialización debido a la menor susceptibilidad al ataque de patógenos (García \& Muñoz, 2017). También, Botella-Martínez et al. (2021) al evaluar el efecto del tamaño de partícula en la composición química de harinas de semillas de cacao reportaron humedades promedias de grano de cacao rangos de 6,79 a 7,05\% comprendidos en lo obtenido en las semillas de cacao provenientes de Copallín y La Peca. Además, el promedio global de número de granos, 100 gramos de granos seleccionados, fue de 95,8, valor similar a lo reportado por Vera et al. (2019); estos valores, Fuentes (2016) lo atribuye a un manejo adecuado de prácticas agrícolas y procedencia del material genético durante la selección de árboles elites de cacao. 
REVISTA DE LA UNIVERSIDAD DEL ZULIA. $3^{a}$ época. Año $13 \mathrm{~N}^{\circ}$ 36, 2022 Jhon More Calderón et al. // Caracterización física y sensorial de almendras de plantas de cacao ... 56-69 DOI: http://dx.doi.org/10.46925//rdluz.36.04

Tabla 1. Características físicas de almendras de cacao de árboles elites

\begin{tabular}{|c|c|c|c|c|}
\hline Distrito & Codificación & $\begin{array}{l}\text { Humedad } \\
(\%)\end{array}$ & $\begin{array}{c}\text { Número de } \\
\text { granos en } 100 \\
\text { gramos }\end{array}$ & $\begin{array}{c}\text { Peso de } \\
\text { grano (IG) g }\end{array}$ \\
\hline Copallín & MRC - 08 & 7,0 & 82 & 1,4 \\
\hline Copallín & MRC - 05 & 7,2 & 106 & 1,5 \\
\hline Copallín & ABL-03 & 7,3 & 86 & 1,4 \\
\hline Copallín & ABL-01 & 7,0 & 114 & 1,2 \\
\hline Copallín & MRC-06 & 7,7 & 98 & 1,3 \\
\hline Copallín & VVA-02 & 7,3 & 94 & 1,2 \\
\hline Copallín & SBL-03 & 7,3 & 106 & 1,4 \\
\hline Copallín & FPG-02 & 7,3 & 69 & 1,4 \\
\hline Copallín & MRC-02 & 7,3 & 106 & 1,4 \\
\hline Copallín & MRC-10 & 7,1 & 106 & 0,9 \\
\hline Copallín & ABL-02 & 6,5 & 109 & 0,9 \\
\hline Copallín & SCF-01 & 6,7 & 93 & 1,8 \\
\hline Copallín & DTM-02 & 6,7 & 74 & 1,4 \\
\hline .Copallín & MRC-04 & 7,3 & 62 & 1,6 \\
\hline La Peca & JBB-04 & 7,2 & 126 & 1,6 \\
\hline La Peca & JBB-02 & 7,0 & 78 & 1,2 \\
\hline La Peca & JCC-06 & 7,4 & 88 & 1,2 \\
\hline La Peca & SCHB-Ol & 7,4 & 95 & 1,2 \\
\hline La Peca & ARC-02 & 7,7 & 120 & 1,3 \\
\hline La Peca & JCC-02 & 7,3 & 103 & 1,4 \\
\hline
\end{tabular}

De los resultados obtenidos, 02 muestras de árboles élites de Copallín presentaron índice de grano por debajo de 1,0 (ABL-02 y MRC-10 fueron 0,9), pero en 18 muestras de Copallín y La Peca (90\% de los árboles elites) fue mayor a 1,0 similar a lo reportado Vera et al. (2019) quienes mencionan que el índice debe ser superior al 1,0 tanto desde el punto de vista filogenético e industrial, descartando valores menores a 1,0. También estos valores de índice de grano (0,9 a 1,2) están dentro de los encontrados por Marca \& Maldonado (2018) en cacao nacional boliviano, siendo valores de índices productivos. Las 20 muestras presentaron un índice de grano promedio de 1,34 similar a lo hallado en árboles élites con valores de 1,50 a 1,88 según Nieves \& Angulo (2017) y de 1,19 a 2,0 según Vera et al. (2019). También, Fuentes 
REVISTA DE LA UNIVERSIDAD DEL ZULIA. $3^{a}$ época. Año $13 \mathrm{~N}^{\circ}$ 36, 2022 Jhon More Calderón et al. /// Caracterización física y sensorial de almendras de plantas de cacao ... 56-69 DOI: http://dx.doi.org/10.46925//rdluz.36.04

(2016) refiere que valores altos de IG están atribuidos a condiciones edáficas del suelo y un buen manejo agronómico, evidenciándose en mayores rendimientos de frutos del árbol.

Respecto a los resultados obtenidos en la prueba de corte, las muestras de almendras ABL-01 y JCC-02 tuvieron 45 granos bien fermentados que representaron un 90\%. Asimismo, en 11 muestras los granos bien fermentados tuvieron valores en el rango de 64 al 90\% (Figura 1), lo cual está dentro lo reportado por Fuentes (2016) que establece valores entre 62,2\% y 75\%; esto significaría que el material recolectado evidenció árboles elites con una alto porcentaje de almendras fermentadas, lo cual influye directamente en la calidad sensorial (Vallejo et al. 2018).

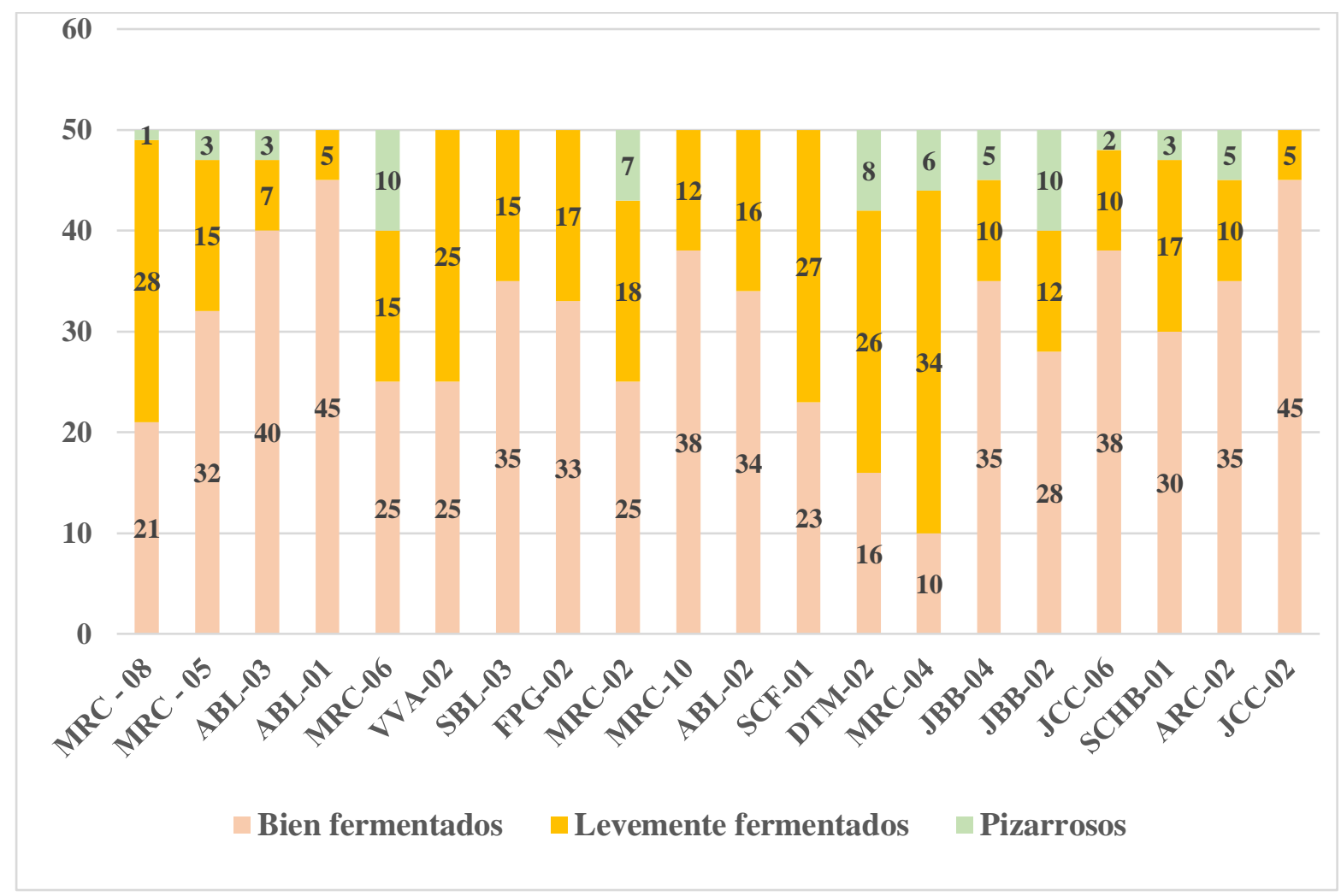

Figura 1. Prueba de corte en almendras de cacao elite

En la Figuran 2 se presentan los atributos básicos. El atributo dulce de la almendra tuvo una mayor percepción en ABL-0l $(4,0)$ en comparación a la ausencia de dulzura percibido en la SCF-01, provenientes de Copallín; en cuanto a la amargura, en las almendras de seis árboles elites de Copallín solo se percibieron dicho atributo (ABL-02, DTM-02, FPG-02, MRC-04, MRC-10 y SCF-01), pero no en las almendras con origen La Peca; la astringencia tuvo valores comprendidos entre 1,0 y 4,4 de puntos, y en tres muestras presentaron puntajes mayores a 
REVISTA DE LA UNIVERSIDAD DEL ZULIA. $3^{a}$ época. Año $13 \mathrm{~N}^{\circ}$ 36, 2022

Jhon More Calderón et al. // Caracterización física y sensorial de almendras de plantas de cacao ... 56-69

DOI: http://dx.doi.org/10.46925//rdluz.36.04

4,0 (ABL-02, MRC-04, SCF-01); paralelamente, la acidez estuvo en un rango de puntuación de 1,0 a 4,2 (FPG-02 mostró el puntaje más alto). Sin embargo, los puntajes en todas las plantas de cacao elite guarda relación con lo reportado por Solórzano et al. (2015) y con los valores intermedios de acidez, astringencia y amargura de cacaos venezolanos (Ramos et al. 2013).

2.2. Determinación del perfil sensorial de atributos básicos y específicos de cacao nativo elite

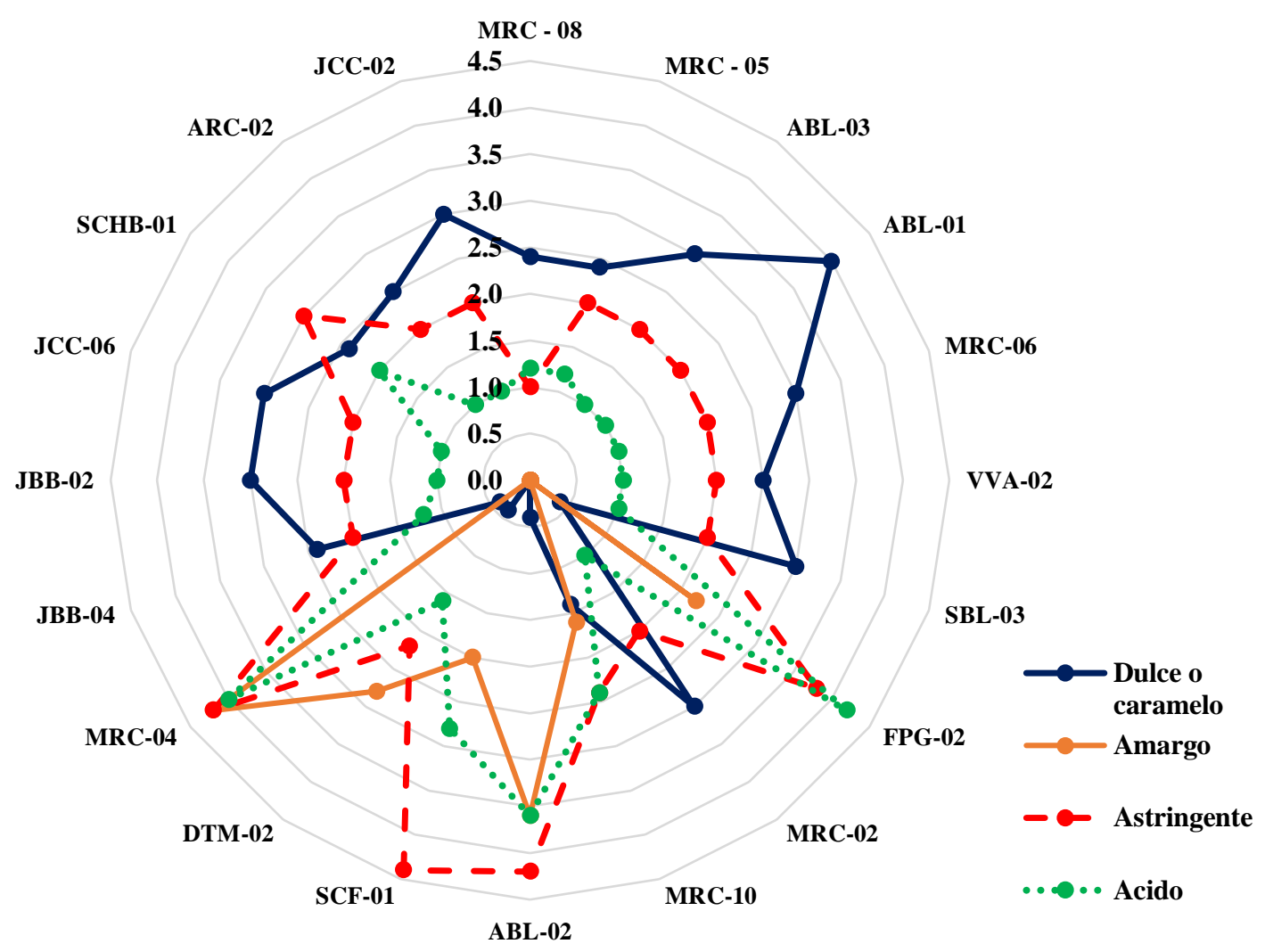

Figura 2. Perfil sensorial de los atributos básicos de las almendras de cacao elite

Respecto a los atributos específicos evaluados, en la Figura 3 se aprecia que sensorialmente el cacao tuvo calificaciones en un rango de 2,6 a 6,0 donde las almendras del árbol SBL-03 (Copallín) fue del sabor a cacao característico y ABL03 (Copallín) resaltó respecto a los demás en su sabor a nueces. En los granos de cacao, su atributo floral, la mayor puntación fue para MRC-08 (Copallín); en general dicho atributo en los cacao elites según su procedencia su puntaje fluctuó desde 1,0 a 4,0; al respecto, es importante lograr una mayor intensidad sensorial percibida de los atributos específicos (cacao, frutal, herbal, floral, y 
REVISTA DE LA UNIVERSIDAD DEL ZULIA. $3^{a}$ época. Año $13 \mathrm{~N}^{\circ}$ 36, 2022

Jhon More Calderón et al. /// Caracterización física y sensorial de almendras de plantas de cacao ... 56-69

DOI: http://dx.doi.org/10.46925//rdluz.36.04

nuez); los aromas y sabores específicos son generados por reacciones enzimáticas durante la poscosecha del grano de cacao y pueden influenciar en la calidad organoléptica (Ramos et al. 2013; Marca \& Maldonado, 2018). También, se evidencia que los atributos de floral, frutal y cacao son percibidos con mayor intensidad al analizar sensorialmente el cacao que según Vallejo et al. (2018) son atributos específicos requeridos para obtener un chocolate fino.

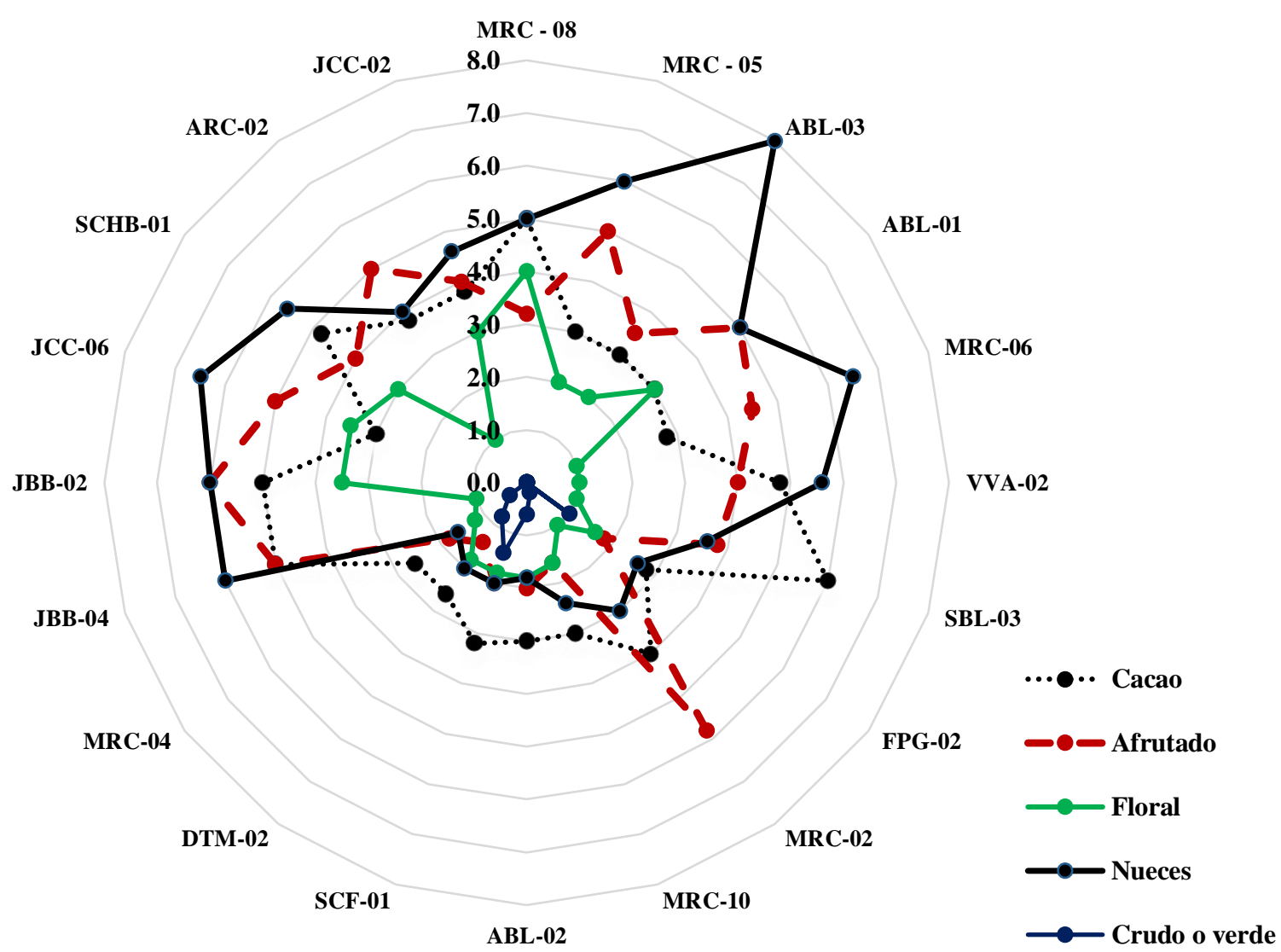

Figura 3. Perfil sensorial de los atributos específicos de las almendras de cacao élite

En la Figura 4 se observa que los atributos sensoriales dulce, afrutado y nueces se correlacionan positivamente más en el primer componente principal $(\mathrm{PCl})$; es así que las almendras de cacaotales provenientes de La Peca resaltan más en los atributos floral, nuez y dulce y en menor nivel en sabor a cacao; paralelamente las almendras provenientes de Copallín se acentúan sus atributos de sabores básicos (amargo, ácido y astringente) y en sabor a cacao. Las almendras de la muestra DTM-02 tuvo una mayor correlación para los atributos de crudo y amargo; mientras la muestra MRC-10 reportó una tendencia a una mejor astringencia y acidez. El primer componente principal ( $\mathrm{PCl}$ ) representó el 66,1\% de la 
REVISTA DE LA UNIVERSIDAD DEL ZULIA. $3^{a}$ época. Año $13 \mathrm{~N}^{\circ}$ 36, 2022

Jhon More Calderón et al. /// Caracterización física y sensorial de almendras de plantas de cacao ... 56-69

DOI: http://dx.doi.org/10.46925//rdluz.36.04

varianza total, valor por debajo al componente principal de los atributos sensoriales $(85,4 \%)$ encontrado por Machado et al. (2018). Por otra parte, respecto a los atributos que se correlacionaron más con el $\mathrm{PCl}$ fueron los sabores de dulce $(0,39)$, afrutado $(0,35)$ y nueces $(0,34)$; estos valores son similares a lo reportado por Solórzano et al. (2015) respecto a los atributos frutales, y nueces que tuvieron una alta correlación; sin embargo, no coincidieron con los valores de atributos cacao, amargor y nuez. Solórzano et al. (2015) y Coronado-Jorge et al. (2016) y Vallejo et al. (2018) mencionan que el mayor amargor en las almendras es debido a una fermentación deficiente, mientras que la acidez sería señal de la presencia de ácidos (volátiles y no volátiles) y en varias almendras de árboles elites (Figura 3) donde se ha presentado sabores a cacao, afrutado, floral, amargor y astringencia moderados.

\section{Conclusiones}

En cuanto a la calidad física del grano, la humedad que contiene las almendras cacao de los árboles elites (6,5\% a 7,7\%) están dentro de lo requerido (menor a 8\%), los números de granos en 100 gramos con valor promedio global $(95,8)$ indican un manejo agrícola y un origen del material genético adecuados; y referente al índice de grano (1,20 a 1,80) en 18 árboles elites son valores aceptables para comercializarlos. Así mismo, los valores de la prueba de corte expresan un alto porcentaje de almendras fermentadas lo que influye directamente en la calidad sensorial.

La calidad sensorial en las almendras de los árboles elite estudiados respecto a sus atributos básicos de amargor, astringencia y acidez obtuvo una alta intensidad de percepción sensorial en las almendras de las plantas de cacao MRC-04 (Copallín). Por otra parte, las almendras provenientes de La Peca resaltan más en los atributos floral, afrutado y dulce respecto a Copallín, donde es más para los atributos básicos. Por último, los atributos básicos y específicos de estas almendras elites son muy requeridos para la producción de chocolate con fino aroma. 


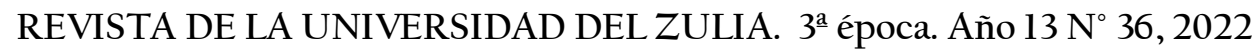

Jhon More Calderón et al. // Caracterización física y sensorial de almendras de plantas de cacao ... 56-69

DOI: http://dx.doi.org/10.46925//rdluz.36.04
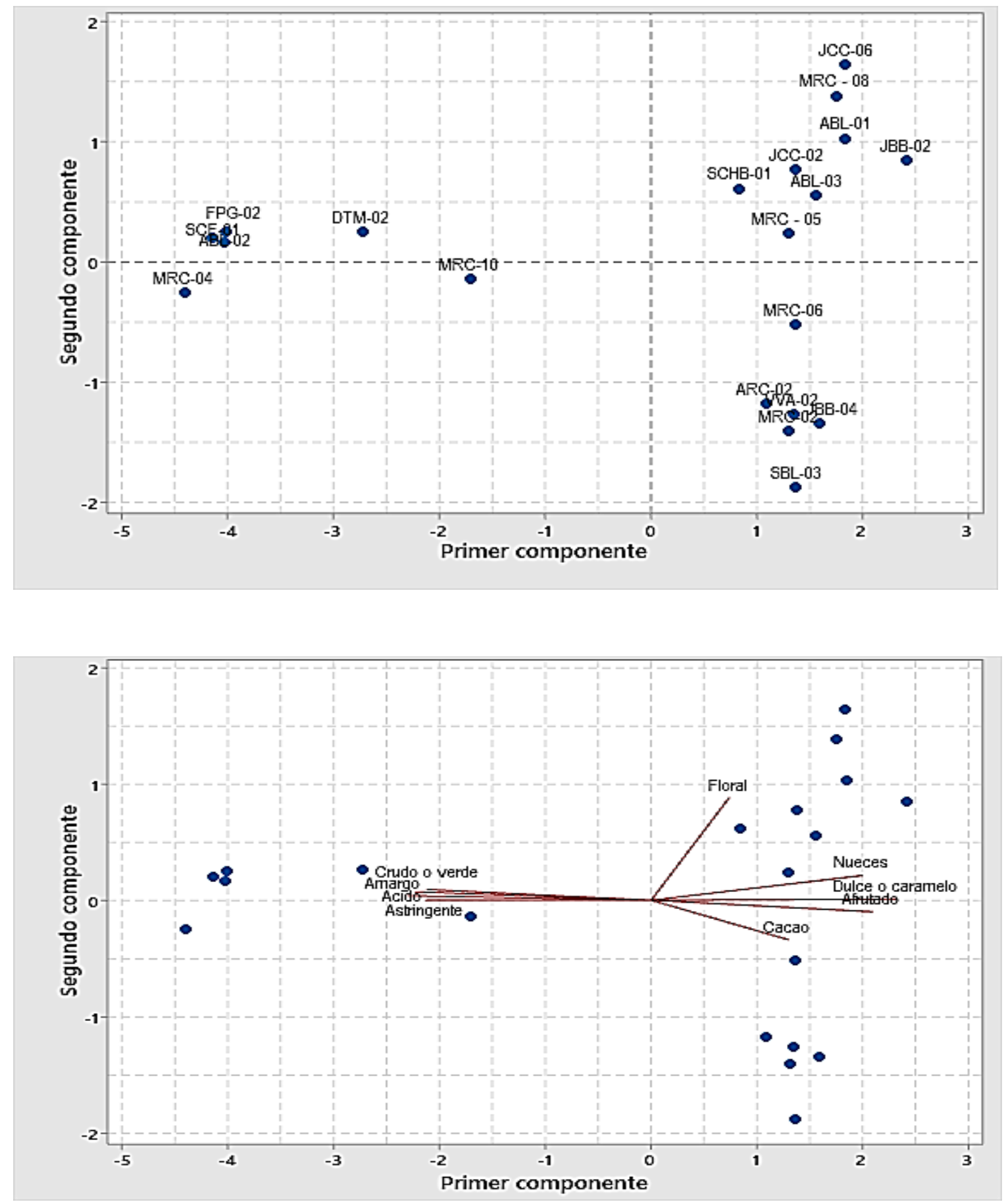

Figura 4. Análisis de componentes principales de los atributos sensoriales en almendras de cacao elites

\section{Referencias}

Ballesteros, W. (2011). Caracterización morfológica de árboles elite de cacao (Theobroma cacao L.) en el municipio de Tumaco, Nariño, Colombia [Tesis de Maestría, Universidad de Nariño]. http://biblioteca.udenar.edu.co:8085/atenea/biblioteca/86414.pdf.

Barrientos, L. D. P., Oquendo, J. D. T., Garzón, M. A. G., \&® Álvarez, O. L. M. (2019). Effect of 
REVISTA DE LA UNIVERSIDAD DEL ZULIA. $3^{a}$ época. Año $13 \mathrm{~N}^{\circ}$ 36, 2022

Jhon More Calderón et al. /// Caracterización física y sensorial de almendras de plantas de cacao ... 56-69

DOI: http://dx.doi.org/10.46925//rdluz.36.04

the solar drying process on the sensory and chemical quality of cocoa (Theobroma cacao L.) cultivated in Antioquia, Colombia. Food Research International, 115, 259-267. https://doi.org/10.1016/j.foodres.2018.08.084.

Botella-Martínez, C., Lucas-Gonzalez, R., Ballester-Costa, C., Pérez-Álvarez, J., FernándezLópez, J., Delgado-Ospina, J., Chaves-López, C., \& Viuda-Martos, M. (2021). Ghanaian cocoa (Theobroma cacao L.) bean shells coproducts: Effect of particle size on chemical composition, bioactive compound content and antioxidant activity. Agronomy, (ll) 401. https://doi.org/10.3390/agronomyll020401 www.mdpi.

Castro-Alayo, E., Idrogo-Vásquez, G., \& Siche, R., Cárdenas-Toro, F. (2019). Formación de precursores de compuestos aromáticos durante la fermentación del cacao criollo y forastero. Heliyon 5. e01157. https://doi.org/10.1016/j.heliyon.2019.e01157.

Chire, G. C., Verona, P. A., \& Guzmán, J. H. (2016). Cambios en el color durante el beneficio del grano de cacao (Theobroma cacao L.) peruano procedente de Piura. Ciencia e Investigación, 19(1), 29-34. https://revistasinvestigacion.unmsm.edu.pe/index.php/farma/article/view/13625.

Coronado-Jorge, M., Cerna, A., Santander, W. E., \& Paucar, W. (2016). Influencia de la diversidad varietal y el estado de madurez sobre la calidad sensorial de granos de Cacao (Theobroma cacao L.) producidos en la región de San Martín. Hilea Amazónica, 1(2), 138-156. http://revistas.unsm.edu.pe/index.php/Ia/article/view/ACl7.

Fuentes, O. R. (2016). Evaluación de la calidad de las almendras del cacao de ascendencia nacional de árboles elites seleccionados en la zona de Vinces y Palenque-Ecuador [Tesis de Grado, Universidad de Guayaquil].

http://repositorio.ug.edu.ec/bitstream/redug/18526/1/tesis\%20de\%20orly\%20fuentes\%20lo renty.pdf.

García, L. A., \& Muñoz, W. A. (2017). Diagnóstico de los parámetros físicos del cacao en centros de acopio en la zona de influencia de los valles Carrizal-Chone [Tesis de Grado, Escuela Superior Politécnica Agropecuaria de Manabí-Manuel Felix López]. http://repositorio.espam.edu.ec/xmlui/handle/42000/533.

Guimac, L. (2017). Caracterización fisicoquímica y organoléptica del cacao criollo nativo (Theobroma cacao L.) de las parcelas cacaoteras de APROCAM [Tesis de Grado, Universidad Nacional Toribio Rodríguez de Mendoza de Amazonas]. http://repositorio.untrm.edu.pe/handle/UNTRM/1348.

Intriago, F. G., Talledo, M. V., Cuenca, G. J., Macías, J. R., Álvarez, J. R., \& Menjívar, J. C. (2019). Evaluación del contenido de metales pesados en almendras de cacao (Theobroma cacao L.) durante el proceso de beneficiado. Revista de Producción, Ciencias e Investigación, 3(26), 17-23. https://doi.org/10.29018/issn.2588-1000vol3iss26.2019ppl7-23.

Marca, J., \& Maldonado, C. (2018). Caracterización morfológica de cacao nacional boliviano (Theobroma cacao L.) en Sapecho, Apthapi 4(2), 1082-1088. chrome- 
REVISTA DE LA UNIVERSIDAD DEL ZULIA. $3^{a}$ época. Año $13 \mathrm{~N}^{\circ}$ 36, 2022

Jhon More Calderón et al. /// Caracterización física y sensorial de almendras de plantas de cacao ... 56-69

DOI: http://dx.doi.org/10.46925//rdluz.36.04

extension://efaidnbmnnnibpcajpcglclefindmkaj/viewer.html?pdfurl=http $\% 3 \mathrm{~A} \% 2 \mathrm{~F} \% 2 \mathrm{Fww}$

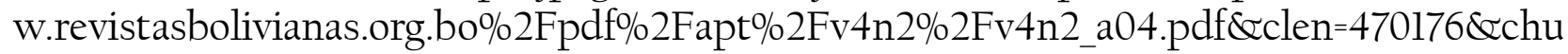
nk=true.

Moreno-Martínez, E., Gavanzo-Cárdenas, O., \& Rangel-Silva, F. (2019). Evaluación de las características físicas y sensoriales de licor de cacao asociadas a modelos de siembra. Ciencia y Agricultura, 16(3), 75-90. https://doi.org/10.19053/01228420.vl6.n3.2019.9890.

Machado, L., Ordoñez, C. M., Angel, K., Guaca, L., \& Suárez, J. C. (2018). Organoleptic quality assessment of Theobroma cacao L. in cocoa farms in northern Huila, Colombia. Acta Agronómica, 67(1), 46-52. https://doi.org/10.15446/acag.v67nl.66572.

Nieves, I. G. E., \& Angulo, L. B. M. (2017). Prospección y caracterización de árboles promisorios de cacao (Theobroma cacao L.) en el rio Onzole. Dominio de las Ciencias, 3(Extra 3), 100-125. https://dialnet.unirioja.es/servlet/articulo?codigo=6102846.

Peláez, Pedro. P., Guerra, S., \& Contreras, D. (2016). Changes in physical and chemical characteristics of fermented cocoa (Theobroma cacao L.) beans with manual and semimechanized transfer, between fermentation boxes. Scientia Agropecuaria, 07(02), 111-119. https://doi.org/10.17268/sci.agropecu.2016.02.04.

Qin, X.W., Lai, J.-X., Tan, L.-H., Hao, C.Y., Li, F.P., He, S.Z., \& Song, Y.-H. (2017). Characterization of volatile compounds in criollo, forastero, and trinitario cocoa seeds (Theobroma cacao L.) in China. International Journal of Food Properties, 20(10), 2261-2275. https://doi.org/10.1080/10942912.2016.1236270.

Quevedo, J. N., Romero, J. A., \& Tuz, I. G. T. (2018). Calidad físico química y sensorial de granos y licor de cacao (Theobroma cacao L.) usando cinco métodos de fermentación. Revista Científica Agrosistemas, 6(1), 115-127. https://aes.ucf.edu.cu/index.php/aes.

Ramos, G., L, González, N., Zambrano, A., \& Gómez, A. (2013). Olores y sabores de cacaos (Theobroma cacao L.) venezolanos obtenidos usando un panel de catación entrenado. Revista Científica UDO Agrícola $114 \quad 13$ (1), 114-127. chromeextension://efaidnbmnnnibpcajpcglclefindmkaj/viewer.html?pdfurl=http $\% 3 \mathrm{~A} \% 2 \mathrm{~F} \% 2 \mathrm{Fww}$ w.bioline.org.br\%2Fpdf\%3Fcgl3014\&eclen=555128.

Ramos, A.; Gómez, M.; Machado-Sierra, E.; Aranguren, Y. (2020). Caracterización fenotípica y genotípica de cultivares de cacao (Theobroma cacao L.) de Dibulla, La Guajira, Colombia. Cienc. Tecnol. Agropecuaria, 21 (3): el557. https://doi.org/10.21930/rcta.vol21 num3 art:1557.

Solórzano, E., Amores, F., Jiménez, J. J., Nicklin, C., \& Barzola, S. (2015). Comparación sensorial del cacao (Theobroma cacao L.) nacional fino de aroma cultivado en diferentes zonas $\begin{array}{lllll}\text { del Ecuador. Ciencia } \quad y \quad \text { Tecnología., } & 8(1), & 37-47 .\end{array}$ https://dialnet.unirioja.es/descarga/articulo/5319282.pdf. 
REVISTA DE LA UNIVERSIDAD DEL ZULIA. $3^{a}$ época. Año $13 \mathrm{~N}^{\circ}$ 36, 2022 Jhon More Calderón et al. /// Caracterización física y sensorial de almendras de plantas de cacao ... 56-69 DOI: http://dx.doi.org/10.46925//rdluz.36.04

Vallejo, C. A., Loayza, G. L., Morales, W., \& Vera, J. (2018). Sensory profile of genotypes of cocoa (Theobroma cacao L.) un the parish of Valle Hermoso-Ecuador. Revista ESPAMCIENCIA para el agro, 9(2), 103-113. https://dialnet.unirioja.es/servlet/articulo?codigo=7020076.

Vera, J. F., Véliz, B. M., \& Herrera, N. L. (2019). Physical quality of almonds in twenty-one interconnectional crosses of cocoa (Theobroma cacao L.) in Ecuador. Universidad y Sociedad, ll(2), 402-408. http://rus.ucf. edu.cu/index.php/rus.

Wickramasuriya, A. M., \& Dunwell, J. M. (2018). Cacao biotechnology: Current status and future prospects. Plant Biotechnology Journal, 16(1), 4-17. https://doi.org/10.1111/pbi.12848. 